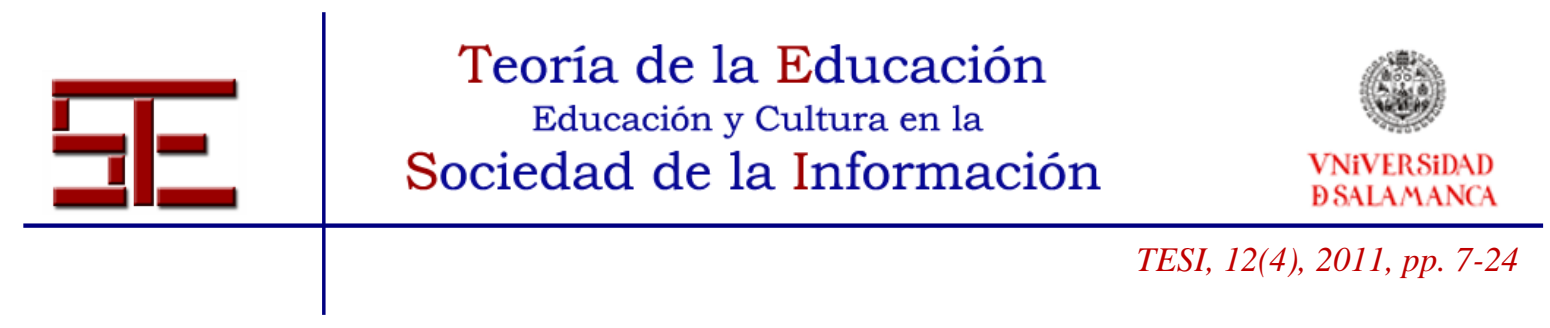

\title{
ECOSISTEMAS DE FORMACIÓN BLENDED-LEARNING PARA EMPRENDER Y COLABORAR EN LA UNIVERSIDAD. VALORACIÓN DE LOS ESTUDIANTES SOBRE LOS RECURSOS
}

Resumen: En este trabajo recogemos los resultados obtenidos en un proyecto de innovación que forma parte de una investigación longitudinal sobre ecosistemas de formación que estamos desarrollando en colaboración con la Universidad del Algarve y otros centros de innovación (Innobridge, Suiza y Valnalón, España). En esta ocasión presentamos la valoración que sobre los recursos empleados realizan los estudiantes en diversos títulos y cursos de la Facultad de Formación del Profesorado y Educación de la Universidad de Oviedo. La muestra está formada por 363 estudiantes y la metodología es cuantitativa. Los resultados indican una positiva valoración del contexto de enseñanza-aprendizaje semipresencial y los recursos tecnológicos y humanos empleados porque facilitan la gestión del conocimiento de manera colaborativa entre los agentes educativos implicados así como por la participación de profesionales externos que proyectan el ecosistema de formación en el entorno laboral.

Palabras clave: Educación Superior; metodología docente; estudiante universitario; ecosistema de formación.

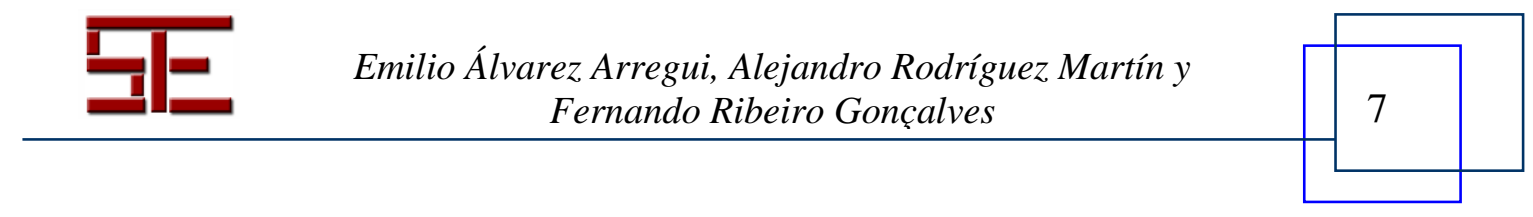




\title{
ECOSYSTEM-LEARNING BLENDED TRAINING FOR ENTERPRISES AND ASSIST IN THE UNIVERSITY. ASSESSMENT OF STUDENT RESOURCE
}

\begin{abstract}
In this work we present the results obtained in an innovation project that is part of a longitudinal study on ecosystems Training we are developing in collaboration with the University of Algarve and other centers of innovation (Innobridge, Switzerland and Valnalón, Spain). This time we present the assessment of the resources employed students perform in various degrees and courses at the Faculty of Teacher Training and Education at the University of Oviedo. The sample consisted of 120 students and the methodology is quantitative. Results indicate a positive assessment of the context blended learning teaching and technological resources and human resources used because they facilitate knowledge management in a collaborative manner between the educational agents involved and the participation of outside professionals that ecosystem project training the workplace.
\end{abstract}

Keywords: Higher Education; teaching training; student; training ecosystem. 


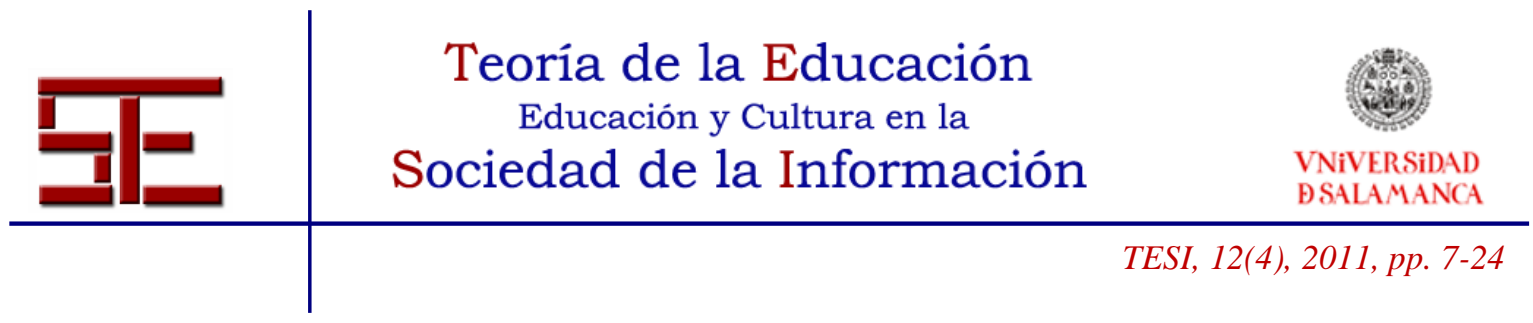

\title{
ECOSISTEMAS DE FORMACIÓN BLENDED-LEARNING PARA EMPRENDER Y COLABORAR EN LA UNIVERSIDAD. VALORACIÓN DE LOS ESTUDIANTES SOBRE LOS RECURSOS
}

Fecha de recepción: 24/10/2011; fecha de aceptación: 03/11/2011; fecha de publicación: 20/12/2011

\author{
Emilio Álvarez Arregui \\ alvarezemilio@uniovi.es \\ Universidad de Oviedo \\ \begin{tabular}{l} 
Alejandro Rodríguez Martín \\
rodriguezmalejandro@uniovi.es \\
\hline Universidad de Oviedo
\end{tabular} \\ Fernando Ribeiro Gonçalves \\ fgonc@ualg.pt \\ Universidade do Algarve
}

\section{1.- INTRODUCCIÓN}

La Sociedad y la Universidad se van reinterpretando en cada momento histórico por las variables que se entrelazan y concurren situacionalmente, de ahí que las misiones de estas organizaciones varíen en base a las peculiaridades de los contextos, de los grupos humanos implicados, de las políticas que se promuevan, de los recursos disponibles, de la adecuación de los modelos de gobernanza a las prescripciones y de las demandas internas y externas.

Actualmente, las Instituciones de Educación Superior están diseñando escenarios y estrategias diferenciales de aprendizaje interconectadas en función de sus objetivos, posibilidades y perfil de los usuarios adaptando gradualmente su Misión y Estrategia a las demandas de una Sociedad de la Información que aspira a convertirse en una Sociedad del Conocimiento.

La Universidad de Oviedo ha apostado decididamente por esta vía promoviendo un Campus de Excelencia a través del plan "Ad Futurum" que se enmarca en la Estrategia Universidad 2015 y que tiene como objetivo la modernización, la especialización y la internacionalización del sistema universitario español. El Campus de Excelencia se ha presentado en distintos foros como motor de un Ecosistema Asturiano del

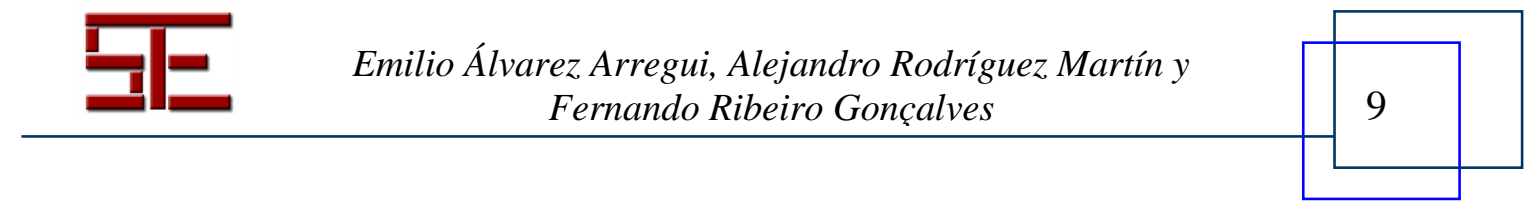




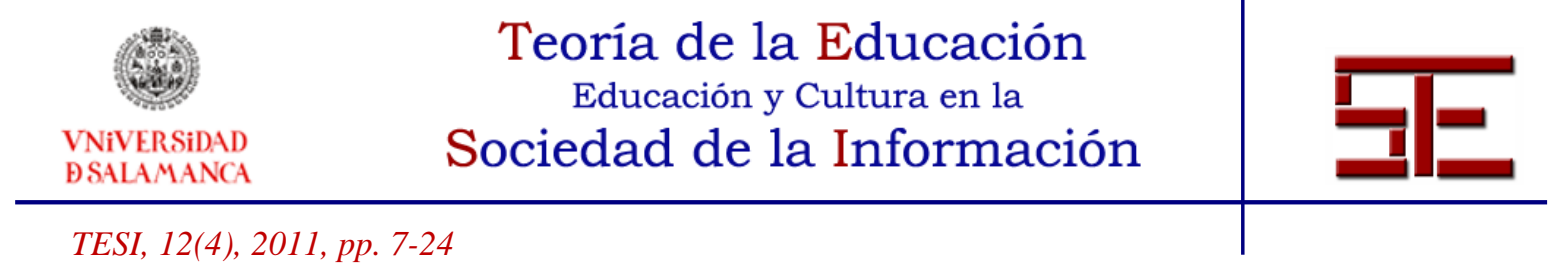

Conocimiento, comprometido con la sociedad, inclusivo y abierto a los ciudadanos en cualquier momento de su trayectoria vital y profesional.

Los modelos de aprendizaje que se promueven conjugan con mayor o menor éxito lo presencial y lo virtual desde una perspectiva ecosistémica bajo la suposición de que este planteamiento es una buena alternativa de trabajo y supone un reto para las Facultades en el caso de la educación pone a prueba la sabiduría didáctica, organizativa, curricular, evaluadora e investigadora con el objeto de favorecer la promoción de una cultura emprendedora que ligue el aprendizaje con la innovación, la creatividad y el mercado laboral.

En este contexto las modalidades de formación presencial, semipresencial o virtual no pueden presentarse como excluyentes o como complementarias, sino que debemos explorar nuevas posibilidades para integrarlas de manera flexible, coherente y equitativa en los entornos profesionales y sociales rompiendo los esquemas disciplinares habituales, los espacios y los tiempos. En este marco es donde se integran las investigaciones que venimos desarrollando sobre los ecosistemas de formación.

\section{2.- APROXIMACIONES A LOS ECOSISTEMAS DE FORMACIÓN}

Los ecosistemas de formación blended learning (aprendizaje mixto) se vienen mostrando como una de las mejores alternativas para responder a las incertidumbres que rodean lo educativo, lo curricular, lo laboral, lo organizativo y el desarrollo profesional en momentos de cambio; de ahí que nos acercaremos a ellos desde distintas perspectivas.

En primer lugar, la palabra "ecosistema", tal y como recoge en el diccionario de la Real Academia Española, procede de la raíz griega "eco-oiko", que significa "morada" o "ámbito vital", y de la palabra latina -systema- que hace referencia a un conjunto de cosas que relacionadas entre sí ordenadamente contribuyen a determinado objeto. En conjunto y adoptando una perspectiva biológica podemos definir la palabra "ecosistema" como una comunidad de seres vivos (biocenosis) cuyos procesos vitales se relacionan entre sí y se desarrollan en función de los factores físicos de un mismo ambiente (biotopo).

Esta definición con un marcado carácter biológico enfatiza tres aspectos, "seres vivos", "relación-desarrollo" y "factores físicos", que, en nuestra opinión, son claves en la propuesta de ecosistema de formación que planteamos porque se promueven redes de

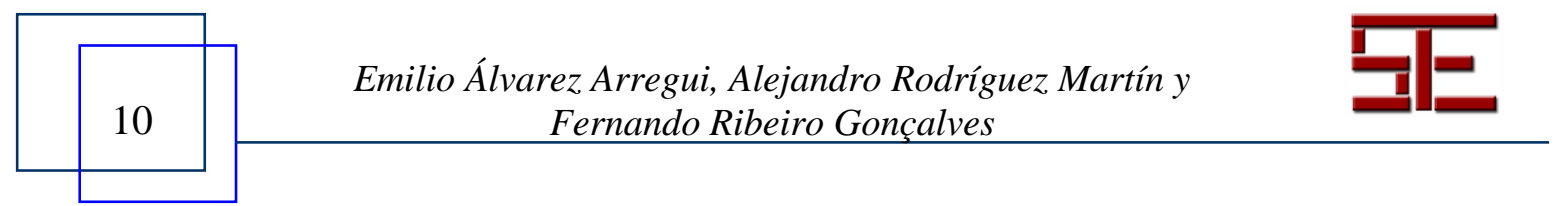




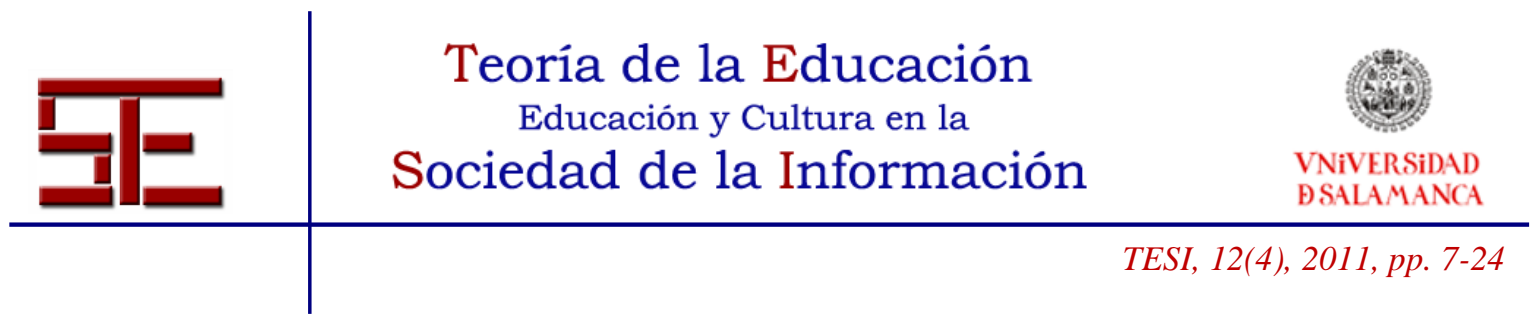

colaboración entre estudiantes, profesorado y profesionales que desarrollan aprendizajes en un contexto presencial y virtual atendiendo a los condicionantes situacionales. Es, pues, un concepto multidimensional (Pickett \& Cadenasso, 2002) que incorpora una visión holística al integrar componentes físicos y humanos, las relaciones e interacciones que se generan, las estructuras de poder, el liderazgo, la creatividad, el espíritu emprendedor y el contexto.

Esta perspectiva ecosistémica ha sido abordada desde otros enfoques y campos de conocimiento. Destacan Bertalanffy (1982), a través de su teoría de los sistemas; Bunge (1980), desde la pertinencia del análisis de sistemas para la investigación ecológica; Bronfenbrenner (1987), quien plantea el desarrollo como el resultado de la interacción entre una persona en crecimiento y las singularidades del medio ecológico dinámico en el que está inmerso; Tikunoff (1979) y Habermas (1989), que se adentran en la dimensión comunicativa; Doyle (1977), que se centra en los procesos de negociación, o Gimeno Sacristán (1988) en el currículo.

Este planteamiento ecosistémico choca frontalmente en educación con las visiones reduccionistas y disciplinares del currículum porque va más allá de la consideración aislada de las personas, los objetivos, los contenidos, la evaluación o las experiencias, ya que todos estos elementos deben ser considerados junto a las relaciones e interacciones que se generan internamente así como con los otros ecosistemas con los que interacciona constantemente, lo que conlleva adoptar simultáneamente un enfoque global, local y situacional cuando se quieren buscar soluciones al complejo problema de la educación (Álvarez Arregui y Rodríguez Martín, 2011).

Los modelos ecosistémicos de formación se convierten así en una alternativa viable para dar respuestas a las demandas de una sociedad compleja en constante cambio y, desde ellos, se abordan aquellas cuestiones a las que se está dando una gran importancia en los entornos socioeducativos y laborales, caso de las habilidades para gestionar el propio aprendizaje; disponer de elementos y procesos de enseñanza-aprendizaje flexibles; comunicar y compartir los conocimientos sin barreras lingüísticas y culturales; enriquecer los aprendizajes con la incorporación y participación de profesionales de empresas, administraciones, ONGs, etc., y, finalmente, incorporar el espacio virtual para favorecer un ecosistema que dé respuesta a tales demandas (Visser, 1999; Pilotti y Sedita, 2005; Witherspoon, 2006; Álvarez Arregui, 2010; Álvarez Arregui y Rodríguez Martín, 2011 a, b y c).

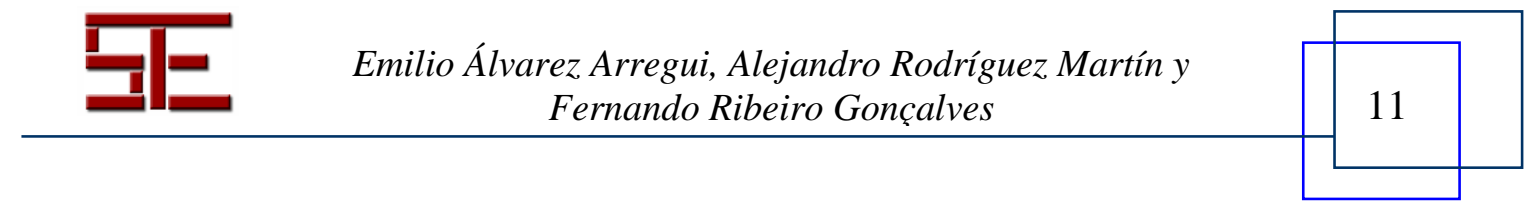




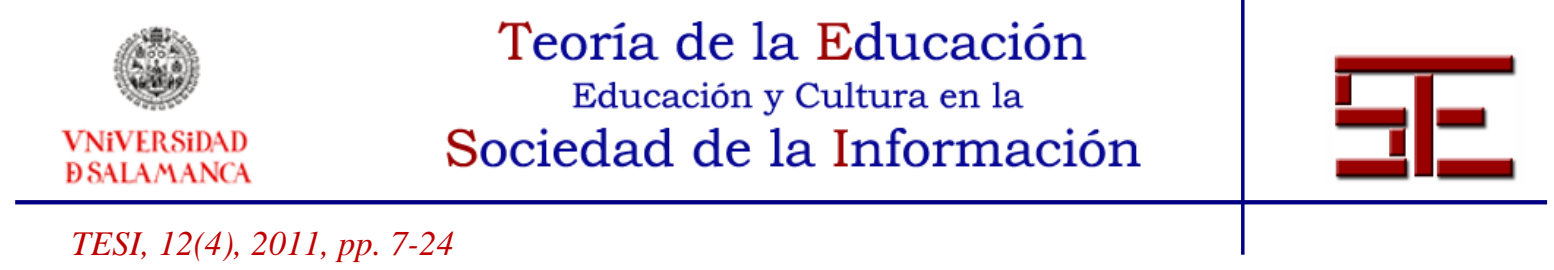

Gült y Chang (2009) han revisado distintos enfoques desarrollados bajo esta perspectiva planteando que cuando se quieren potenciar vías de colaboración a partir del establecimiento de relaciones dinámicas entre los miembros de las comunidades de aprendizaje es aconsejable el desarrollo de distintos "ecosistemas holísticos basados en enfoques de aprendizaje e-learning". De entre otros, cabe destacar el Ecosistema modular (Dimitrov, 2001); el Ecosistema del conocimiento (Shrivastava, 1998); el Ecosistema e-learning de trabajo y aprendizaje (Wilkinson, 2002); el Ecosistema elearning (Brodo, 2006), y el Ecosistema e-learning basado en tecnologías y sistemas de gestión y apoyo al aprendizaje (Ismail, 2001). Gült y Chang (2009) trabajan en un enfoque que denominan "Learning Ecosystem" (LES), donde integran a todos los participantes en el proceso de aprendizaje prestando especial atención a los agentes educativos y sociales y a la generación de redes.

Estos modelos ecosistémicos incorporan entre sus componentes un diseño de aprendizaje (Maneschijn, 2005) los recursos humanos (profesorado, estudiantes, agentes educativos, etc.), una tecnología de apoyo (Cowley et al., 2002; Rodríguez Martín y Escandell, 2004), una formación para el desarrollo de capacitación en competencias básicas (Kulathuramaiyer y Maurer, 2008) y aplicaciones de la web 2.0 (Shimaa, Nasr y Helmy, 2011).

Estos antecedentes, junto con el contexto que nos proporciona el Campus de Excelencia Internacional de la Universidad de Oviedo, son los referentes del Ecosistema de Formación blended learning que hemos planteado para el desarrollo profesional del profesorado universitario y de los estudiantes incorporando un cambio de orientación de la metodología docente a través de cinco fases: el diagnóstico del entorno de intervención, el diseño del contexto de formación, el despliegue e implementación del modelo de aprendizaje, la evaluación para la mejora y la investigación del impacto. El diseño, el proceso de implementación y los resultados se evalúan a través de un sistema polivalente, flexible y continuado donde se tienen en cuenta los objetivos de partida, pero también los imprevistos, dadas las múltiples situaciones que emergen cuando se pretende promover la creatividad y la emprendeduría.

\section{3.- OBJETIVO DEL ESTUDIO}

Con la intención de conocer la efectividad del Ecosistema de Formación blended learning, hemos desarrollado un estudio exploratorio con el siguiente objetivo: analizar las percepciones de los estudiantes hacia los recursos empleados en las asignaturas

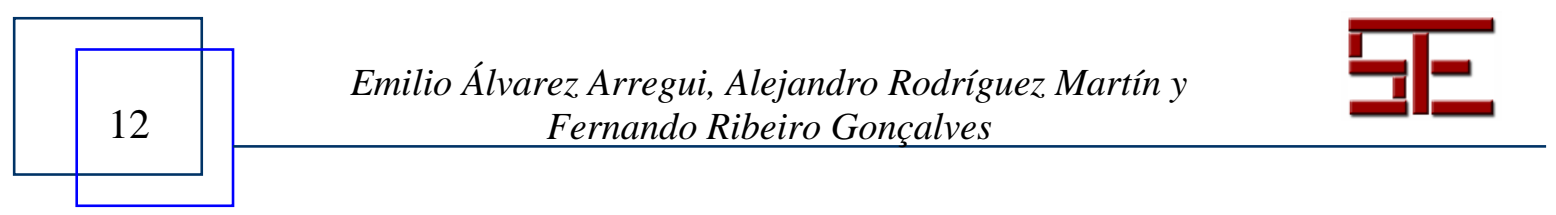




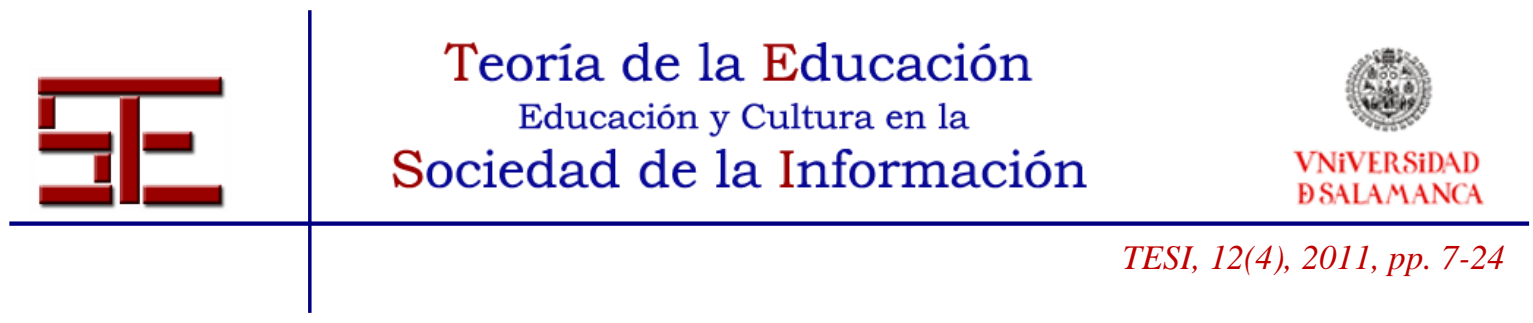

"Organización y Gestión de Centros Educativos" y "Formación y Actualización de la función pedagógica" (Licenciatura en Pedagogía, $3^{\text {er }}$ curso); "Aspectos Didácticos y Organizativos de la Educación Especial. (Maestro, Educación Especial, $3^{\text {er }}$ curso); "Didáctica General" (Grado de Maestro de Educación Primaria, $1^{\text {er }}$ curso) y "Tecnologías de la Información y la Comunicación" (Máster de Formación del Profesorado).

\section{4.- METODOLOGÍA}

El modelo elegido para desarrollar esta investigación ha sido el descriptivointerpretativo ex-post-facto. En este trabajo se presentan los resultados proporcionados a partir del análisis de la dimensión "Recursos del ecosistema" recogidos por un cuestionario de elaboración propia y se incluyen algunos comentarios recogidos en las preguntas abiertas así como en los blogs que utilizamos como apoyo en nuestro trabajo dado que ilustran y complementan los datos cuantitativos.

\section{1.- Participantes}

La población está formada por 2250 estudiantes de la Facultad de Formación del Profesorado y Educación de la Universidad de Oviedo. La muestra seleccionada la componen 363 estudiantes (16\%) matriculados en las asignaturas indicadas anteriormente.

\section{2.- Instrumento y procedimiento}

De acuerdo al objetivo de este estudio y a toda la investigación en la que se integra, se prepararon las distintas dimensiones del cuestionario y se elaboró un cuestionario provisional que se aplicó a una muestra reducida. El instrumento quedó finalmente estructurado en 63 ítems que se valoran desde 1 (totalmente en desacuerdo) hasta 5 (totalmente de acuerdo) y preguntas abiertas en los diferentes apartados. Se estructura en 7 variables de clasificación (sexo, edad, estilo de aprendizaje personal, titulación cursada, otros estudios universitarios cursados, dominio y manejo de aplicaciones informáticas, acceso y uso de herramientas telemáticas). Las dimensiones asociadas al ecosistema de formación fueron las siguientes: docencia, entorno virtual, recursos, evaluación y potencialidad.

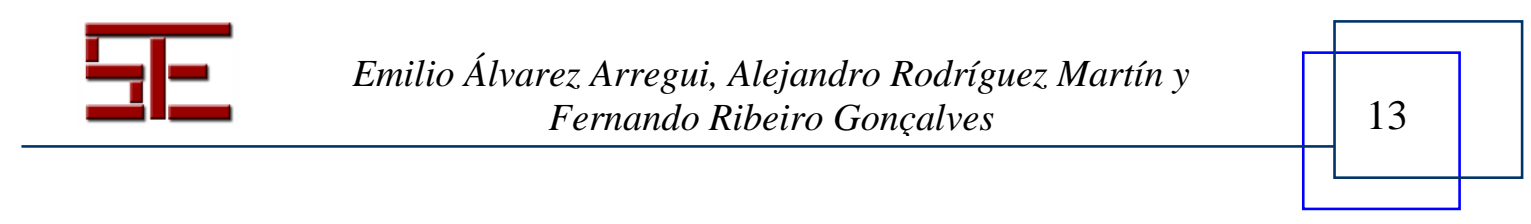




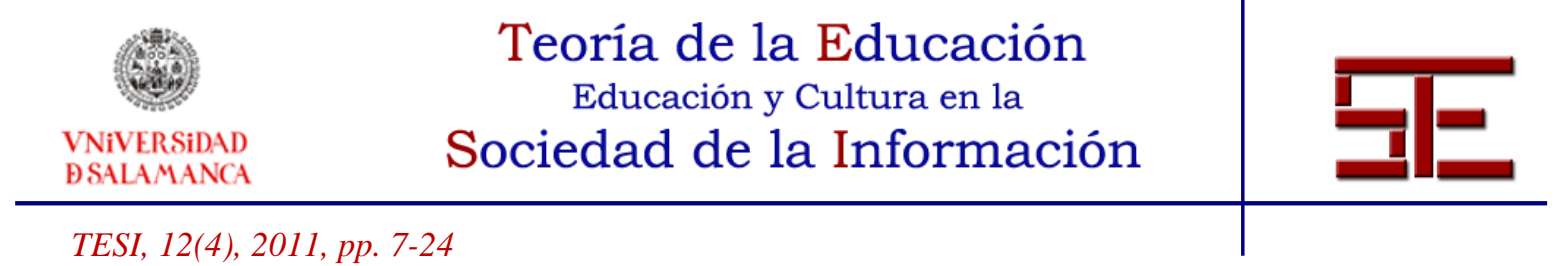

El error muestral es del 5,5\% (95\%) y el nivel de confianza $Z=1,96 ; p=q=0,5(95 \%)$. El nivel de fiabilidad se ha establecido mediante el alfa de Cronbach (.956); la correlación entre formas (,686), Coeficiente Spearman-Brown $(, 814)$ y las Dos mitades de Guttman (,789). La validez se ha determinado a través de tres revisiones internas y otras cinco revisiones de validación por cuatro expertos, tres universidades españolas (Universidad de Oviedo, Universidad de Cantabria y Universidad de Sevilla) y una portuguesa (Universidade do Algarve).

El cuestionario fue aplicado en un único momento por miembros del equipo investigador. Los participantes respondieron de manera individual, voluntaria, sin limitación temporal, e informados de la confidencialidad de sus repuestas.

\section{3.- Análisis de datos}

Las informaciones cuantitativas proporcionadas por los ítems de los cuestionarios fueron tratadas con el programa SPSS 19 través de los siguientes estudios: Análisis de Fiabilidad; Análisis factorial; Análisis de Frecuencias, Diferencias de medias (T-Test para muestras independientes, utilizando los estadísticos $\mathrm{T}$ de Student y el test de Levene para estimar la igualdad de varianzas) y Análisis de Varianza (Anova y test a posteriori de Scheffé con el subprograma oneway).

\section{5.- RESULTADOS}

A continuación se presentan los resultados más significativos respecto a la escala objeto de este estudio (Recursos y herramientas del ecosistema). Inicialmente se recoge el análisis de las frecuencias de respuesta en cada uno de los ítems y, posteriormente, los análisis de diferencias según las variables asignatura y titulación (análisis de varianza). Asimismo los resultados se ilustran con comentarios extraídos de la información cualitativa recogida en los blogs de las asignaturas.

\begin{tabular}{|l|c|c|c|c|c|c|c|}
\hline \multirow{2}{*}{ Ítems } & \multirow{2}{*}{$\overline{\mathrm{X}}$} & \multirow{2}{*}{$\mathbf{D T}$} & \multicolumn{5}{|c|}{ Resultados (\%) } \\
\cline { 5 - 9 } & & & $\mathbf{1}$ & $\mathbf{2}$ & $\mathbf{3}$ & $\mathbf{4}$ & $\mathbf{5}$ \\
\hline 15. Blogs & 3,22 & 1,018 & 3,9 & 17,9 & 38,3 & 24 & 11,6 \\
\hline 16. Correos electrónicos & 3,81 & 1 & 1,7 & 6,9 & 29,8 & 30,6 & 29,5 \\
\hline 17. Foros & 3,14 &, 909 & 2,2 & 19,8 & 46,8 & 21.8 & 8,3 \\
\hline
\end{tabular}

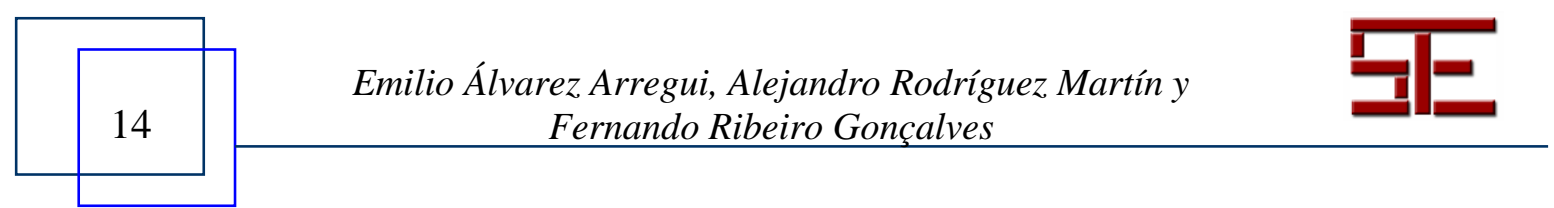




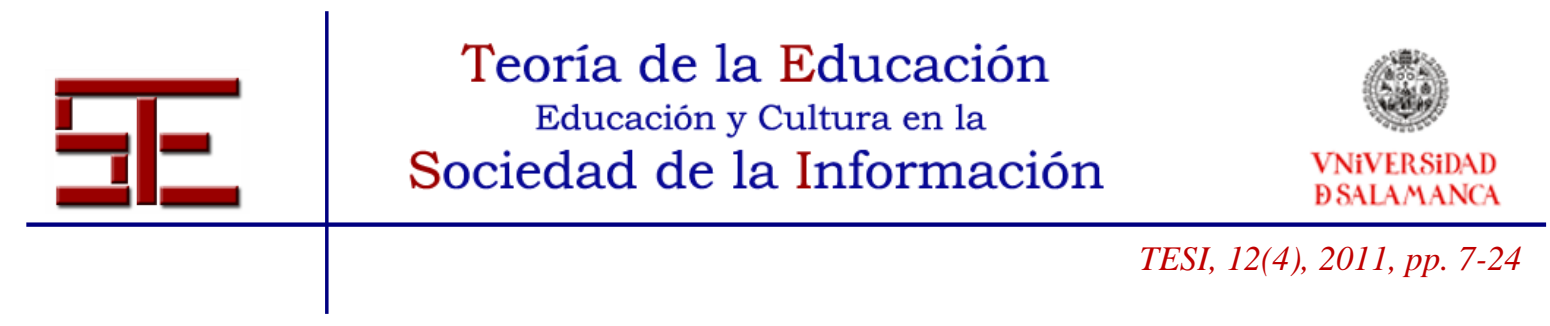

\begin{tabular}{|l|c|c|c|c|c|c|c|}
\hline 18. Chats & 2,83 &, 984 & 8,3 & 24,2 & 41 & 14,3 & 5,2 \\
\hline 19. Glosario & 3,36 &, 955 & 1,9 & 14,9 & 39,9 & 28,9 & 12,7 \\
\hline 20. Wikis & 3,11 &, 897 & 3 & 17,1 & 49 & 19 & 7,4 \\
\hline 21. Videoconferencia & 3,19 & 1,206 & 10,2 & 12,4 & 33,9 & 18,2 & 15,7 \\
\hline 22. Documentos escritos (Word, pdf...) & 3,99 &, 807 & 0 & 1,1 & 29,8 & 38,3 & 30,9 \\
\hline $\begin{array}{l}\text { 23. Documentos en Power Point } \\
\text { (presentaciones profesor y alumnos) }\end{array}$ & 4,07 &, 843 & 0 & 1,9 & 26,4 & 34,4 & 37,2 \\
\hline $\begin{array}{l}\text { 24. Documentos audiovisuales (vídeos, } \\
\text { películas...) }\end{array}$ & 3,86 &, 911 & 1,1 & 3 & 32 & 32,8 & 28,1 \\
\hline $\begin{array}{l}\text { 25. Documentos sonoros (podcasts de } \\
\text { debates, congresos...) }\end{array}$ & 3,40 & 1.014 & 4,1 & 9,9 & 38,8 & 26,4 & 14,6 \\
\hline $\begin{array}{l}\text { 26. Enlaces (páginas web, bases de datos, } \\
\text { artículos...) }\end{array}$ & 3,70 &, 890 & 0 & 6,1 & 39,9 & 30 & 22,6 \\
\hline 27. Charlas y debates con profesionales & 3,47 &, 997 & 2,5 & 10,7 & 37,5 & 26,4 & 16,5 \\
\hline
\end{tabular}

Cuadro 1: Distribución de frecuencias de la escala "Recursos y herramientas"

Los recursos y herramientas integradas en el ecosistema se valoran positivamente, aunque se indica que deben estar más generalizadas en todas las asignaturas, ya que no pueden ser una novedad vinculada a proyectos de innovación o mejora.

Siempre me ha parecido que una canción, un vídeo, una videoconferencia, una experiencia fuera del típico libro o de la charla común, es mucho más pedagógico y provechoso y además se retiene y recuerda mejor (Blog de metodología: estudiante semipresencial).

... nunca me había planteado la utilización de la radio como un recurso pedagógico ya que estamos acostumbrados a las clases magistrales y como mucho al uso de videos o visionar películas... (Blog de metodología: estudiante presencial).

Este tipo de documentación de audio no me resulta idónea para escucharla mirando la pantalla de un ordenador y sólo les saqué jugo cuando me hicieron compañía en el tren. Creo que, de cara a futuros cursos, no estaría mal convertirlos a .mp3 para fomentar esta utilización "portátil" y dosificar su uso... (Blog de metodología: estudiante semipresencial). 


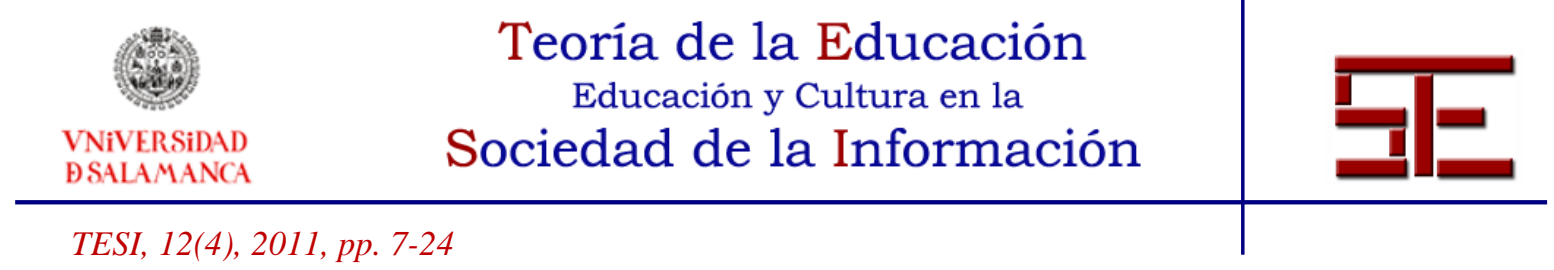

Los estudiantes también conceden una alta valoración a los blogs porque les permiten ajustar la planificación inicial, las metodologías de trabajo presenciales y en red, los tiempos de las tareas prácticas y comprender cómo se gestiona el conocimiento. Las metodologías de trabajo cooperativas permiten hacer visibles nuestras intenciones dado que las dinámicas de grupo se nutren de las aportaciones individuales, de pequeño y gran grupo desde los que se generan debates argumentados y se acomodan los criterios de evaluación en base al grado de dedicación e implicación de los estudiantes.

Me ha sorprendido gratamente la dinámica desarrollada por Emilio para gestionar el conocimiento -individual, de grupo, de aula y en red - ya que nos ha demostrado cómo se incrementan los conocimientos cuando se aúnan perspectivas, se reflexiona en distintos planos y se triangulan las informaciones. Nunca había realizado una dinámica así y debería de generalizarse al resto de asignaturas sustituyendo a los acostumbrados debates que no contribuyen más que a alejar posturas (Blog de metodología: estudiante presencial).

Los imprevistos, cuando derivan en mejoras a partir de la consideración de las opiniones de los estudiantes, son muy bien valorados porque fortalecen el establecimiento de unas relaciones más fluidas y cercanas con los docentes.

Fue muy interesante lo que ocurrió en la sesión teórica del martes cuando, de forma fortuita, falló el aparataje tecnológico. Este hecho obligó a Emilio a cambiar de estilo docente, sirviéndose del encerado e improvisando. Esta situación creó una "dinámica conversacional" que me mantuvo mucho más implicado en la explicación. La falta de guión provocó una mayor atención de Emilio respecto a los mensajes de retroalimentación del grupo -tanto implícitos como explícitos- y generó un alto nivel de atención y de comprensión. Esta situación me hace proponer la alternancia de las exposiciones generales de los temas con apoyo y sin apoyo de diapositivas ya que redundará en un mejor aprovechamiento por nuestra parte (Blog de metodología: estudiante presencial).

El glosario (VM: 3,96), las wikis (VM: 3,66) y la videoconferencia (VM: 3,56) tienen valoraciones por encima de la media y, aunque tienen porcentajes inferiores al resto de herramientas, pueden explicarse por el desconocimiento previo del recurso y por las

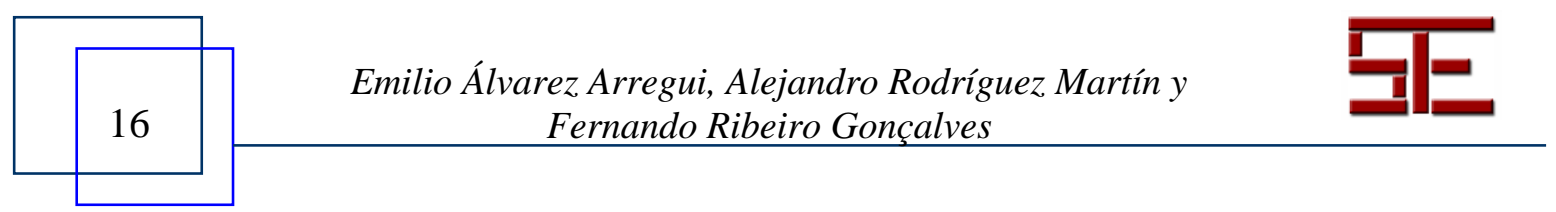




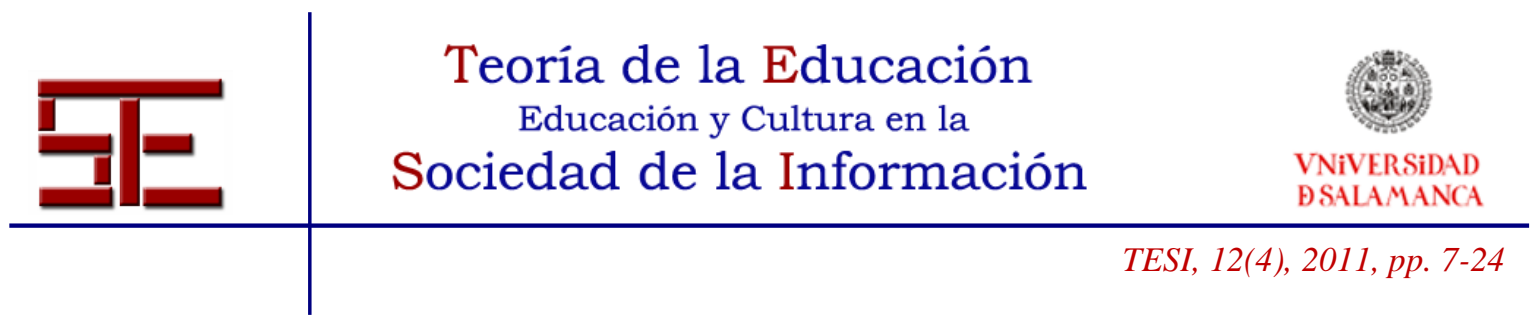

dificultades técnicas que hubo en algunas ocasiones, lo que exigía asesoramiento y mayor tiempo de dedicación, lo que era muy valorado en algunas épocas del curso.

... lo primero que quiero decir es que tenéis que ver el video que nos ha subido Emilio en la entrada, es muy bueno. Yo nunca había utilizado una wiki,.... Sin embargo, después de ver el video lo entiendo perfectamente, es divertido y nos pone un ejemplo en vez de explicar teóricamente lo que es. Esta herramienta es muy buena para potenciar el trabajo en equipo y la comunicación entre todos (Blog de metodología: estudiante no presencial).

Los documentos de consulta, ampliación y debate se valoran como pertinentes. En algunos casos, se apuntan como una sobreinformación, aunque también destacan su interés para ir generando bases de datos reutilizables en otras asignaturas o en el futuro. Los mejor valorados son los documentos audiovisuales incluidos en los blogs (VM: 5.40) o cuando tenían enlaces directos a ellos (VM: 4.90).

... el comportamiento de los monos ejemplifica bastante bien el hecho que ocurre en algunas organizaciones, ya que cuando llegan personas nuevas con ganas de innovar o aportar ideas diferentes se encuentran con que su actitud es vista por las personas que ya trabajan allí como una provocación, puesto que les parece mejor quedarse como están por simple comodidad o por miedo a no saber cómo afrontar nuevas experiencias... (Blog de metodología: estudiante semipresencial).

$\mathrm{Al}$ alumnado le parece interesante el tener acceso a las presentaciones utilizadas por el profesor, los especialistas, los grupos de trabajo y los estudiantes (VM: 5,10). Destacan los comentarios de los alumnos no presenciales en este punto, ya que el planteamiento adoptado les permite tener un seguimiento puntual de la asignatura y debatir en los blogs sus impresiones.

La pizarra digital fue muy interesante, me parecería muy útil que enseñaran a los alumnos a utilizarla, ya que es un recurso del que apenas se le saca partido... (Blog de contenidos: estudiante presencial).

La exposición del vídeo "A golpe de tacón" ha sido un acierto, pone de manifiesto aspectos sociales y organizacionales que nos llevaron a debatir la situación de la mujer a lo largo de la historia (Blog de contenidos: estudiante presencial).

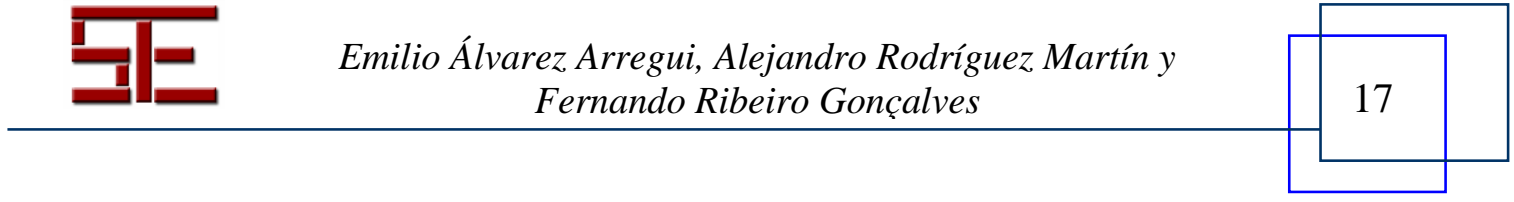




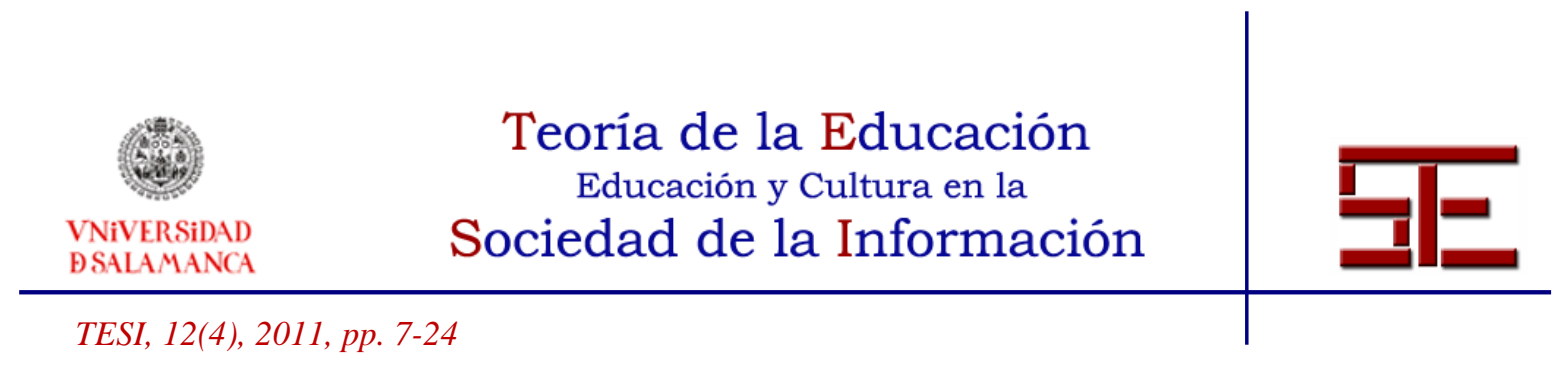

\begin{tabular}{|c|c|c|c|c|c|c|c|c|c|c|c|}
\hline \multirow{2}{*}{$\begin{array}{c}\text { Ítem } \\
\mathrm{s}\end{array}$} & \multicolumn{2}{|c|}{ Lic. Pedagogía } & \multicolumn{2}{|c|}{ Maestr Ed. Esp. } & \multicolumn{2}{|c|}{ Grado Maestro } & \multicolumn{2}{|c|}{ Máster } & \multirow{2}{*}{$\mathbf{F}$} & \multirow{2}{*}{ Sig. } & \multirow{2}{*}{ Contraste } \\
\hline & $\overline{\bar{X}}$ & Sx & $\overline{\bar{X}}$ & Sx & $\overline{\bar{X}}$ & $\mathbf{S x}$ & $\overline{\bar{X}}$ & $\mathbf{S x}$ & & & \\
\hline 15. & 3.23 & 1.046 & 3.55 & .946 & 2.75 & .725 & 3.38 & 1.101 & 8,009 &, 000 & $(1>2)$ \\
\hline 17. & 2.94 & .862 & 3.75 & .926 & 2.97 & .548 & 3.17 & 1.037 & 14,067 & ,000 & $(2>3,1)$ \\
\hline 22. & 3.86 & 7.18 & 4.47 & .808 & 3.72 & .750 & 4.08 & .839 & 13,376 & 000 & $(3<1,2)$ \\
\hline 23. & 3.95 & .816 & 4.62 & .651 & 3.65 & .766 & 4.21 & .837 & 19,998 & ,000 & $(3<1,2)$ \\
\hline 24. & 3.81 & .726 & 4.53 & .755 & 3.12 & .955 & 4.06 & .806 & 36,629 & ,000 & $(3<1,2)$ \\
\hline 25. & 3.20 & .903 & 4.11 & 1.033 & 2.89 & .937 & 3.61 & .884 & 22,233 &, 000 & $(3<1,2)$ \\
\hline 26. & 3.61 & .841 & 4.37 & .782 & 3.04 & .572 & 3.90 & .851 & 34,558 &, 000 & $(3<1,2)$ \\
\hline 27. & 3.50 & 1.004 & 3.74 & .964 & 2.85 & .797 & 3.75 & .946 & 13,463 & ,000 & $(4>3)$ \\
\hline
\end{tabular}

Atendiendo a la variable "titulación", encontrarnos que, siendo las medias bastantes altas, los estudiantes del Grado de Maestro en Educación Primaria son los que, en líneas generales, obtienen puntuaciones inferiores en la mayoría de los ítems de la escala. Observamos que los estudiantes del Máster de secundaria tienen puntuaciones superiores en el ítem 27, ya que las charlas con profesionales fueron una constante durante el desarrollo de la asignatura y el trabajo colaborativo que se desarrolló fue más intenso. En conjunto los datos nos permiten observar que, aunque se plantean diferencias significativas, la tendencia de respuesta es positiva en cuanto a la utilización de los recursos tecnológicos empleados en el ecosistema desarrollado en las asignaturas de los cuatro títulos analizados.

\section{6.- CONCLUSIONES}

Los resultados obtenidos avalan los recursos utilizados en el modelo planteado. También nos hace comprender que tenemos que conceder mayor importancia a la fase de diagnóstico inicial cuando diseñamos el contexto de aprendizaje, porque se burocratizan los procesos, surgen desajustes en la utilización de los recursos existentes, genera frustración cuando hay una baja capacitación en el uso de las herramientas tecnológicas y porque se incrementa el tiempo de trabajo para estudiantes y profesores. Las deficiencias detectadas nos permitirán mejorar el diseño del ecosistema, los recursos utilizados y los sistemas de coordinación interdocente, intrainstitucional e

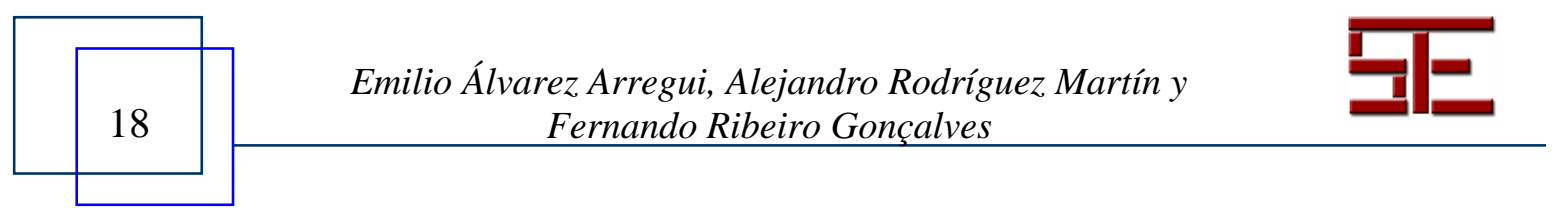




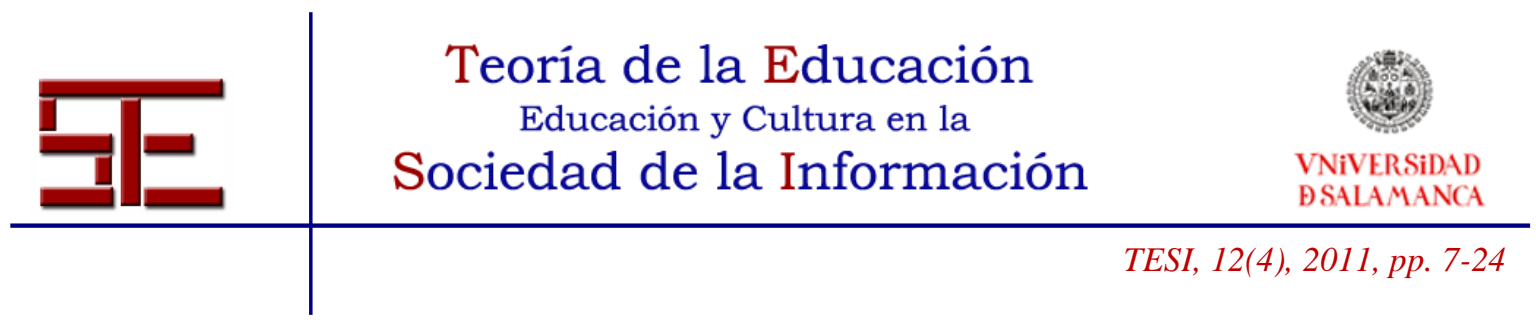

interinstitucional que son referentes básicos para la construcción del Campus de Excelencia Internacional de nuestra Universidad.

Nos parece importante destacar como puntos fuertes del ecosistema el uso de metodologías de trabajo colaborativo (Lavrin y Zelko, 2005) en las sesiones presenciales, que posteriormente se extienden al entorno virtual para que participen los estudiantes no presenciales y los profesionales externos a través de los foros, blogs y chats. Este planteamiento incrementa el grado de satisfacción de los usuarios porque les generó un sentimiento de pertenencia al hacerlos copartícipes independientemente de la modalidad en que cursaron las asignaturas. En cualquier caso, se hace necesario profundizar en el desarrollo de los sistemas de apoyo (tutorías entre iguales), incorporar mejoras en los accesos a conocimientos de calidad (banco de buenas prácticas internas y externas al ecosistema a través del campus virtual y de páginas web) y promover cursos de formación continua inicial y continua especializada a medida que se van incorporando más profesores, estudiantes y recursos tecnológicos en el ecosistema.

Como es lógico el proceso de evaluación se vuelve más complejo porque se hace necesario ir incorporando criterios diferenciadores en función de la modalidad elegida, si bien siempre planteamos como prioritaria la necesidad de potenciar la colaboración en la construcción del ecosistema de formación desde las aportaciones individuales (profesores, alumnos, especialistas, equipos de trabajo...), desde los sistemas de apoyo (tutorías, apoyos de estudiantes) y de trabajo en red (foros, redes, blogs, wikis...), con posibilidad de acceder a conocimientos de calidad (buenas prácticas de los estudiantes...), comentarlos, compartirlos y difundirlos... Por tanto, trabajar en un modelo ecosistémico de formación requiere gestionar pedagógicamente dos espacios, el presencial y el virtual, e interconectarlos a través de múltiples dinámicas de trabajo individual y cooperativo. Los modos de conjugar los distintos elementos concurrentes permiten generar contextos de aprendizaje diferenciado para cada situación de aprendizaje y grupo de alumnos, por lo que debemos ir valorando las distintas alternativas y hacer públicos los resultados para contrastar experiencias y resultados. Este planteamiento requiere apoyarse en un sistema de investigación-acción que debe ser apoyado institucionalmente porque conlleva un proceso de formación continua de todos los agentes implicados y orienta las inversiones en base a las necesidades y a los logros que se van consiguiendo.

Atendiendo a estas cuestiones y a nuestros condicionantes situacionales, hemos promovido un proyecto de innovación aprobado por la Universidad de Oviedo, en el

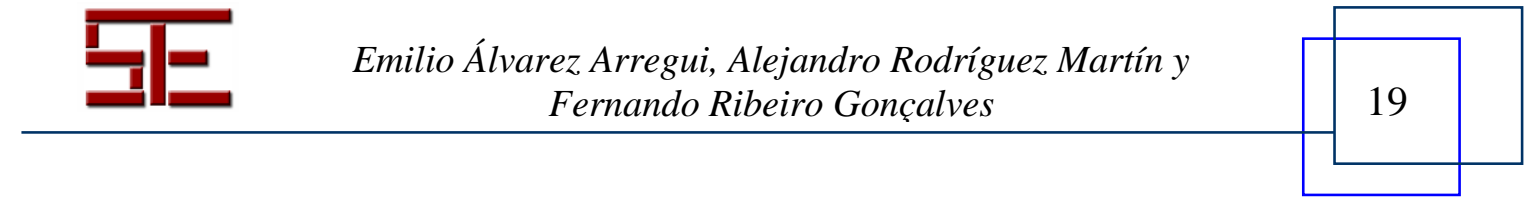




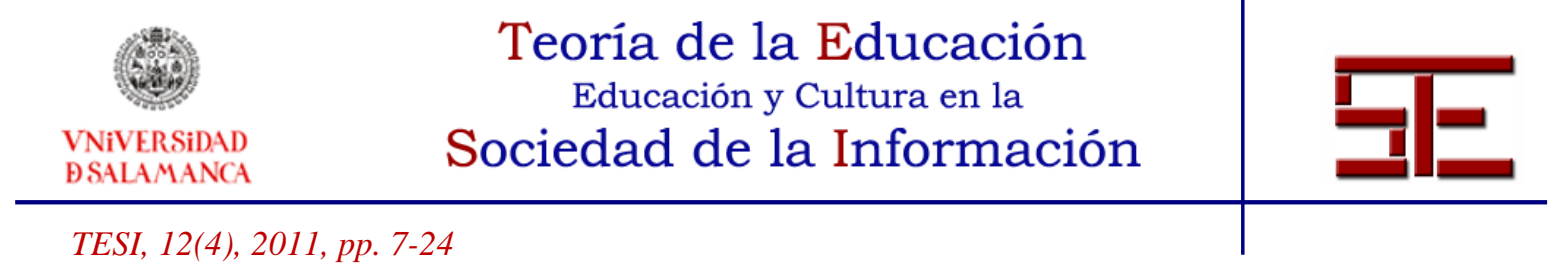

marco del Campus de Excelencia, que tiene por objeto implementar y evaluar un ecosistema de formación blended-learning que experimentarán el profesorado y los estudiantes que cursan las asignaturas de Didáctica, Organización Escolar, Formación y Actualización de la Función Pedagógica, Políticas Educativas y Aspectos Didácticos y Organizativos de la Educación Especial de la Facultad de Formación del Profesorado y de Educación de la Universidad de Oviedo en el presente curso y en el curso 2011/2012. Este proyecto de innovación se ha abierto también a estudiantes y profesores de Administración de Empresas, lo que le confiere un carácter interinstitucional e interdisciplinar que se ha visto reforzado con la concesión de un proyecto de investigación en el que participan profesores de todos los campos de conocimiento de la Universidad de Oviedo.

Atendiendo a estos hechos el modelo ecosistémico de formación se va haciendo más complejo, pero también se dota de un mayor valor añadido porque incorpora los recursos docentes disponibles (plataforma gratuita Moddle, Dropbox, Xing, etc.) en los contextos de aprendizaje que promueve y se nutre del conocimiento interno y externo disponible en el entorno ecosistémico que generamos, ya que contrastamos los datos que vamos obteniendo con otras universidades (Sevilla - España y Algarve - Portugal), recibimos y proporcionamos asesoramiento especializado directo (Ciudad Industrial de Valnalón - España) y atendemos a las recomendaciones que se nos hacen desde un ecosistema suizo de innovación (Innobridge SA) de primera línea mundial. De este modo generamos contextos de aprendizaje donde se promueve:

- la colaboración;

- la autorregulación del aprendizaje;

- la accesibilidad a todos los estudiantes, especialmente, al alumnado con necesidades educativas específicas y/o de otras universidades;

- la generación de criterios fundamentados para incorporar, modificar o rechazar elementos en los diseños de los contextos de aprendizaje (plataformas) en las asignaturas de Grado, en las materias de los másteres, en base al grado de satisfacción de los usuarios y la capacitación adquirida;

- la detección de las necesidades y demandas de formación de los agentes educativos implicados así como de aquellos docentes que quieran utilizar el modelo para poder cubrirlas desde el Instituto de Ciencias de la Educación (ICE) o desde la contratación de profesionales específicos

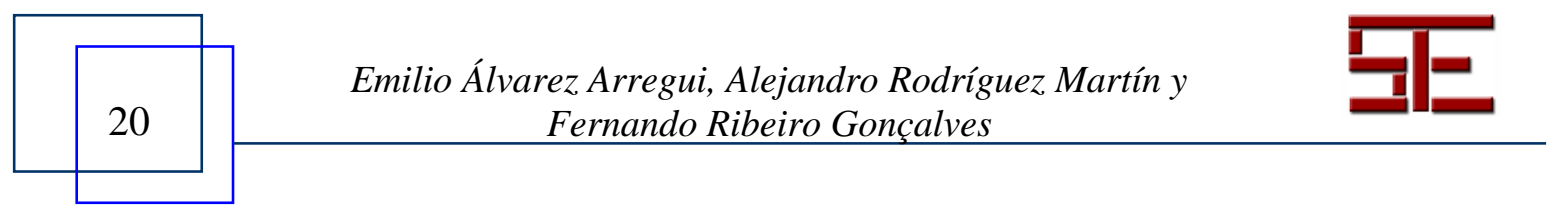




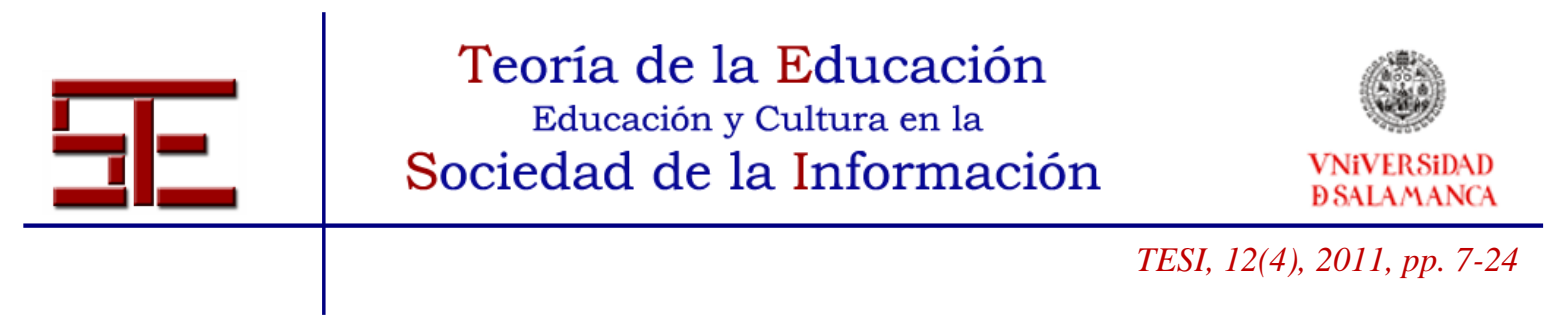

para cuestiones puntuales o desde cursos o seminarios impartidos por el profesorado que desarrolla el modelo;

- la gestión del conocimiento;

- la mejora continua;

- la investigación;

- $\quad \ldots$

Estamos convencidos, que en una Sociedad de la Información con aspiraciones a convertirse en una Sociedad del Conocimiento los modelos ecosistémicos de formación son una buena alternativa que debe seguir explorándose en el ámbito educativo para conjugar la calidad y la equidad, lo académico y lo empresarial y la docencia con la investigación.

\section{BIBLIOGRAFÍA}

Álvarez Arregui, E. (2010a). La universidad ante la excelencia: Posibilidades y límites en períodos de incertidumbre. XI Congreso Internacional de Instituciones Educativas. Cuenca: Universidad de Castilla-La Mancha los días 15, 16 y 17 de diciembre

- (2010b). La universidad ante la excelencia: Posibilidades y límites en períodos de incertidumbre. XI Congreso Internacional de Instituciones Educativas. Cuenca: Universidad de Castilla-La Mancha, los días 15, 16 y 17 de diciembre.

Álvarez Arregui, E. \& Rodríguez Martín, A. (2011a). Aprender a emprender en la Universidad del siglo XXI con Ecosistemas de Formación blended-learning. X Simposio Iberoamericano en Sistemas, Cibernética e Informática (CISCI 2011) en Orlando, Florida, EEUU, los días 19, 20, 21 y 22 de julio de 2011. (Premiada por su calidad, viabilidad y proyección internacional).

- (2011b). Ecosistemas de formación blended learning en el desarrollo profesional del pedagogo. I Congreso sobre el perfil profesional del Pedagogo, celebrado en la Facultad de Ciencias de la Educación (Universidad de Sevilla), los días 8,9 y 10 de junio de 2011.

- (2011c). La tecnología en la mejora de la docencia universitaria: su aplicación en ecosistemas de formación. Comunicación del I Congreso Internacional

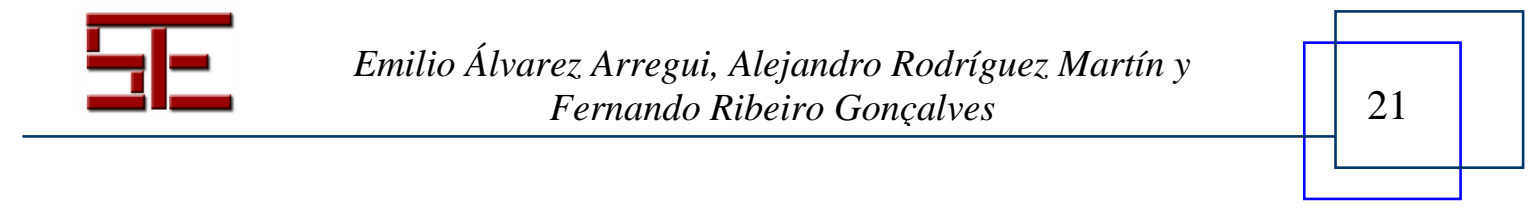




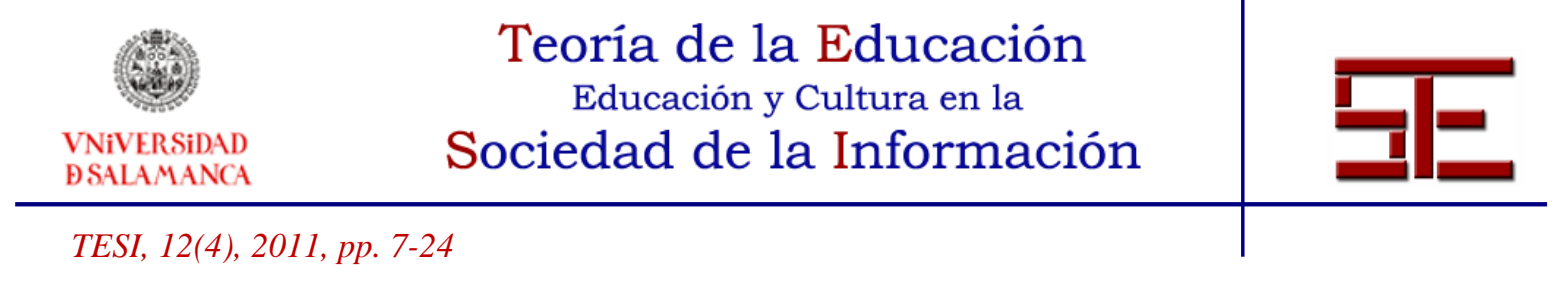

RIAICES en la Universidad del Algarve, Faro, Portugal, los días 22, 23 y 24 de febrero de 2011.

- (2011d). La universidad y el cambio. La innovación de la docencia universitaria como estrategia de adaptación. Comunicación del I Congreso Internacional RIAICES en la Universidad del Algarve, Faro, Portugal, los días 22, 23 y 24 de febrero de 2011.

Bertalanffy, L. (1982). Teoría general de sistemas. Madrid: FCE.

Brodo, J. A. (2006). Today's Ecosystem of e-learning. Trainer Talk, Professional Society for Sales y Marketing Training, vol. 3, N 4, 2006.

Bronfenbrenner, U. (1987). La Ecología del desarrollo humano. Barcelona: Paidós.

Bunge, M. (1980). Epistemología. Barcelona: Ariel.

Cowley, J.; Chanley, S.; Downes, S.; Holstrom, L.; Ressel, D.; Siemens, G. \& Weisburgh, M. (2002). Preparing Students for Elearning. Consultado el día 26 de abril de 2011. Disponible en: http://www.elearnspace.org/Articles/Preparingstudents.htm.

Dimitrov, V. (2001). Learning Ecology for Human and Machine Intelligence. Consultado el día 14 de abril de 2011. Disponible en: http://www.zulenet.com/vladimirdimitrov/pages/LearnEcologyHuman.html.

Doyle, W. (1977). Learning the Classroom Environment: An Edological Analysis, Journal of Teacher Education, $\mathrm{N}^{\mathrm{o}} 28$ (6), 51-55.

Gimeno Sacristán, J. (1988). El currículum, una reflexión sobre la práctica. Madrid: Morata.

Gütl, C. \& Chang, V. (2009). Ecosystem-based Theorical Models for Learning in Environments of th 21st Century. Ijet International Journal of Emerging Technologies in Learning. vol. (7): Hawthorn-Australia, 1-11.

Habermas, J. (1989). Teoría de la acción comunicativa. Madrid: Taurus.

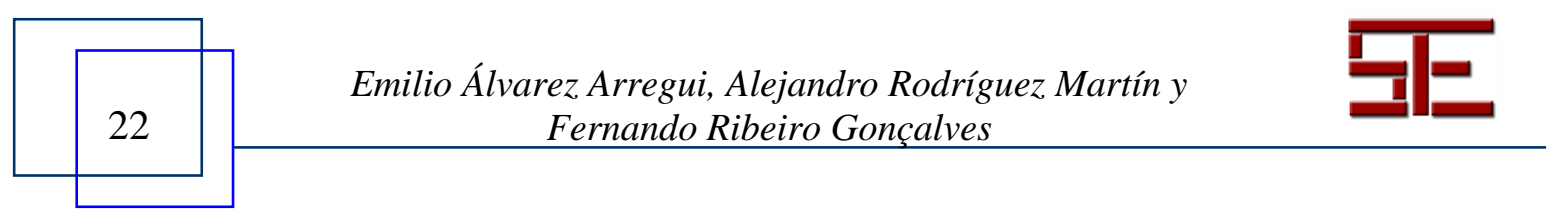




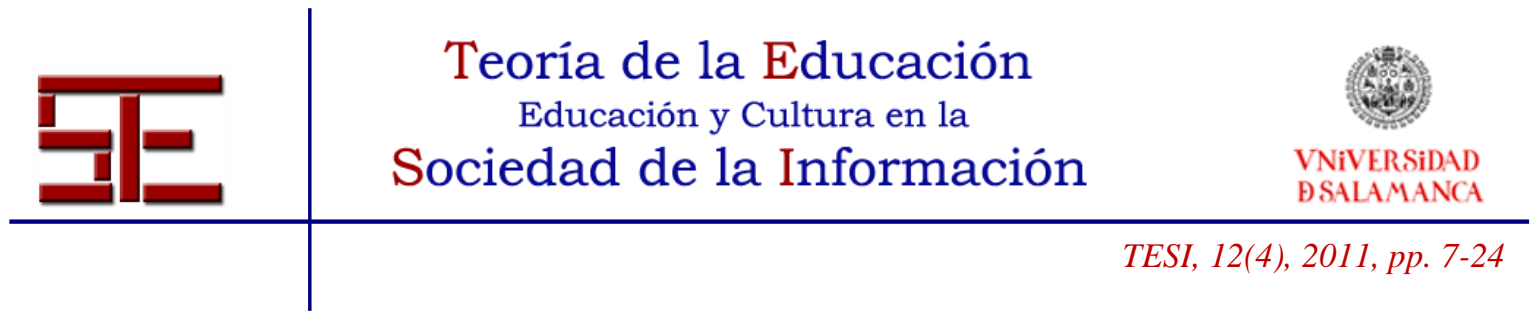

Ismail, J. (2001). The design of an e-learning system Beyond the hype. Internet and Higher Education, vol. 4, Issues 3-4, 329-336.

Kulathuramaiyer, N. \& Maurer, H. (2008). Learning Ecosystems for Dealing with the Copy-Paste Syndrome. Journal of Research in Innovative Teaching, vol. I, Issue I, March, 1-24.

Lavrin, A., Zelko, M. (2005). Knowledge Sharing in Digital Ecosystem for Small and Medium Enterprises IDIMT-2005, 13th Interdisciplinary Information Management Talks, Proceedings Linz, Johannes Kepler Universität Linz, 237252 http://www.sea.uni-linz.ac.at/conferences/idimt2005/session_f.pdf.

Maneschijn, M. M. (2005). The e-learning dome: a comprehensive e-learning environment development model. Consultado el 28 de abril de 2011. Disponible en: http://uir.unisa.ac.za/bitstream/handle/10500/2398/dissertation.pdf?sequence=1

Pickett, S. T. A. \& Cadenasso, M. L. (2002). The Ecosystem as a Multi-dimensional Concept: Meaning, Model, and Metaphor Ecosystems. Ecosystems Journal, New York: Springer, 5, 1-10.

Pilotti, L. \& Sedita, S. R. (2005). Human Capital Development in a Complex Learning System: The Virtuous Interaction between Individuals, Organizations and Communities. Disponible en: from http://ssrn.com/abstract=816884.

Rodríguez Martín, A \& Escandell, Ma . O. (2004). Diversidad y Sociedad de la Información y el Conocimiento: las TIC como herramienta educativa. Anuario de Filosofía, Psicología y Sociología, № 7, 95-106.

Shimaa, O.; Nasr, M. \& Helmy Y. (2011). An Enhanced E-Learning Ecosystem Based on an Integration between Cloud Computing and Web 2.0. IEEE International Conference on Digital Ecosystems and Technologies. Dejeon, Corea, 113-117.

Shrivastava, P. (1998). Knowledge Ecology: Knowledge Ecosystems for Business Education and Training.

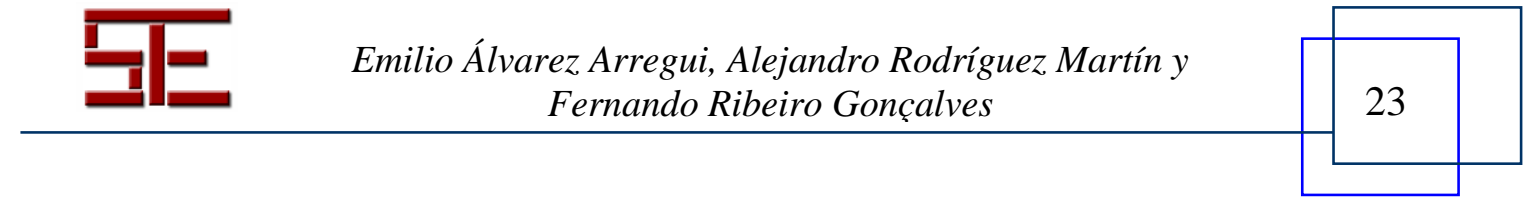


Tikunoff, W. Y. (1979). Context variables of a Tezhing-Learning Event. En D. BENNET and D. McNAMARA, Focus on Teaching. Readings in the observation and conceptualizativon of Teaching. N. Y: Longman.

Visser, J. (1999). Overcoming the underdevelopment of learning: A transdisciplinary view. Annual Meeting of the American Educational Research Association, Montreal, Québec, Canada.

Wilkinson, D. (2002). The Intersection of Learning Architecture and Instructional Design in e-Learning', 2002 ECI Conference on e-Technologies in Engineering Education: Learning Outcomes Providing Future Possibilities, 213-221.

Witherspoon, J. (2006). Building the Academic EcoSystem: Implications of E-Learning. vol. 3, No. 3. http://www.itdl.org/Journal/Mar_06/article01.htm.

Para citar el presente artículo puede utilizar la siguiente referencia:

Álvarez Arregui, E., Rodríguez Martín, A. y Ribeiro Gonçalves, F. (2011). Ecosistemas de formación blended-learning para emprender y colaborar en la universidad. Valoración de los estudiantes sobre los recursos. Revista Teoría de la Educación: Educación y Cultura en la Sociedad de la Información. 12(4), 7-24 [Fecha de consulta: $\mathrm{dd} / \mathrm{mm} / \mathrm{aaaa}]$.

http://campus.usal.es/ revistas_trabajo/index.php/revistatesi/article/view/8523/8609

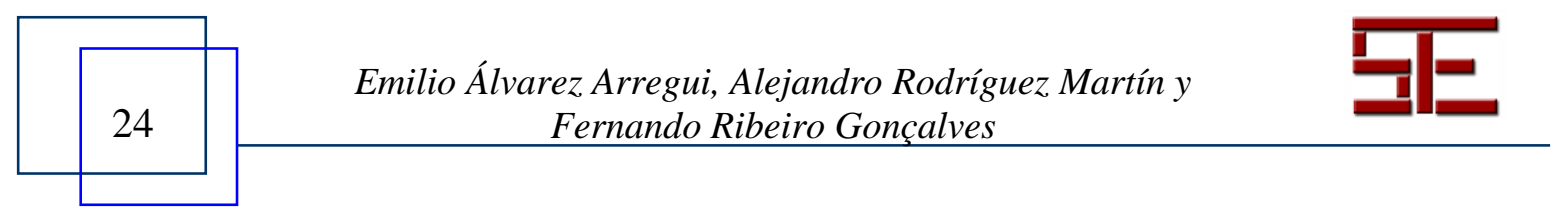

